\title{
A new population-based measure of the economic burden of mental illness in Canada
}

\author{
K-L Lim, PhD (1); P Jacobs, PhD (2); A Ohinmaa, PhD (3); D Schopflocher, PhD (4); CS Dewa, PhD (5)
}

\begin{abstract}
This paper presents a comprehensive measure of the incremental economic burden of mental illness in Canada which incorporates the use of medical resources and productivity losses due to long-term and short-term disability, as well as reductions in health-related quality of life (HRQOL), for the diagnosed and undiagnosed population with mental illness. The analysis was based on the population-based Canadian Community Health Survey Cycle 2.1 (2003). For all persons, we measured all health services utilization, longterm and short-term work loss, and health-related quality of life and their dollar valuations, with the economic burden being the difference in dollar measures between the populations with and without mental health problems. In total, the economic burden was $\$ 51$ billion in 2003. Over one-half was due to reductions in HRQOL. The current accepted practice in economic assessments is to include changes in medical resource use, work loss, and reductions in HRQOL.
\end{abstract}

Key words: Economic burden, cost of illness, mental health, quality of life

\section{Introduction}

Mental illness is a class of conditions which incur a significant use of health care resources, lost productivity, and human suffering. Two recent reports, one by Health Canada $^{(1)}$ and the other by the Senate, ${ }^{(2)}$ have underscored the large economic burden of mental illness in Canada.

Health Canada, in its 2002 Economic Burden of Illness in Canada Report, ${ }^{(3)}$ identified a large economic burden of mental illness from the use of direct government-funded health care services ( $\$ 4.7$ billion in 1998), and the indirect cost of lost productivity due to short- and longterm disability and premature mortality (\$3.2 billion). A 1997 study by Health Canada, ${ }^{(4)}$ using several types of admini- strative and survey data, estimated the economic burden (both direct and indirect costs) of mental disorders in Canada at $\$ 7.8$ billion in 1993 (or $\$ 8.4$ billion in 1998). Stephens and Joubert, ${ }^{(5)}$ using the same data as the 1997 Health Canada study as well as data from certain questions asked in the 1996/1997 National Population Health Survey (NPHS) regarding depression and distress, estimated that direct costs were $\$ 6.3$ billion (which included non-government insured health care services of $\$ 278$ million). The indirect costs of short-term and long-term productivity losses and early death associated with depression and distress were about $\$ 8.1$ billion. The estimated (recalculated) total burden was $\$ 14.4$ billion in 1998 . All these estimates included only mental health services in the direct cost component.
However, the measured economic burden depends on how the concept is defined. We illustrate the concept of economic burden in Figure 1. As can be seen from this figure, economic burden includes both direct and indirect costs. The concept of economic burden, as recently formulated, also includes losses in health-related quality of life (HRQOL) (e.g., increases in pain and suffering) as an essential component. (6-9) $^{(6)}$ These losses from the HRQOL component add to the overall economic burden of illness, and the most recent measures of mental health economic burden incorporate it. ${ }^{(10)}$ The studies on economic burden mentioned above do not include losses in health-related quality of life. In addition, the above-mentioned estimates focus on mental health services costs, rather than on the excess costs of all health services, as the direct cost burden. Persons with mental health problems use both, and so the question of health service use is of considerable importance. In this paper, we report on our attempt to produce such a comprehensive measure for Canada.

The three significant features of our paper are (1) that it includes a measure of the burden of those with undiagnosed mental illness, (2) that the measures are based on the concept of excess costs and losses in outcomes that are attributable to mental illness, not simply measures of gross costs and losses, and (3) that it includes costs for all health services, not just those for mental health.

\section{Author References}

1 Department of Economics, National University of Singapore

2 Faculty of Medicine, University of Alberta and Institute of Health Economics, Alberta

3 School of Public Health, University of Alberta and Institute of Health Economics, Alberta

4 Health Surveillance, Alberta Health and Wellness

5 Centre for Addiction and Mental Health and University of Toronto, Department of Psychiatry, Toronto, Ontario

Correspondence: Arto Ohinmaa, School of Public Health, University of Alberta, 13-103 Clinical Sciences Building, Edmonton, Alberta, Canada T6G 2G3, Tel:

(780) 492-6535, Email: arto.ohinmaa@ualberta.ca 


\section{Methods}

\section{Data}

Data for this analysis came from the 2003 Canadian Community Health Survey (CCHS Cycle 2.1) public-use microdata file. ${ }^{(11)}$ The CCHS is a cross-sectional survey conducted bi-annually by Statistics Canada which provides person-level information on the health status, health system utilization, and health determinants of Canadians living in private occupied dwellings. Sampling weights provided by Statistics Canada were applied to obtain population estimates.

\section{Study population}

The study was confined to the adult population aged 20 and above. We sought to classify individuals into one of three mental health categories (Diagnosed Mental Health Problems, Undiagnosed Mental Health Problems, or No Mental Health Problems) based upon their responses to particular questions selected from the CCHS Cycle 2.1. However, because not all questions were asked of all survey participants, we developed prevalence estimates based upon aggregated data for the three categories, as described in detail below.

For each of the following diagnostic categories, participants were asked whether they had been diagnosed by a health professional as having a major mental health problem in a common content Chronic Disease module. Persons were classified into the category Diagnosed Mental Health Problems if they said yes to any of the following questions: "Do you have schizophrenia?", "Do you have a mood disorder such as depression, bipolar disorder, mania or dysthymia?", or “Do you have an anxiety disorder such as a phobia, obsessive-compulsive disorder or a panic disorder?"

Our method for calculating undiagnosed mental illness follows that of Starkes, et al. ${ }^{(12)}$ but is broader in scope. Persons who had not been classified into the Diagnosed Mental Health category were classified into the Undiagnosed Mental Health category if they had nevertheless met at least one of the following criteria in the past twelve months:

a. In the self-rated mental health question, had reported their mental health as poor;

b. In a module asking about contacts with mental health professionals, had reported 2 or more contacts in person or by telephone to a health professional about emotional or mental health;

c. In a depression screening module consisting of many questions (taken from the Composite Diagnostic Interview Schedule), ${ }^{(13,14)}$ had been scored as having a probability of 0.8 or greater of being a clinical case of depression, or

d. In a module about suicidal ideation or attempts, had indicated having ever seriously considered committing suicide or taking own life.

Only sub-segments of the survey population had been given the optional content questionnaire modules for sub-criteria $b$, $c$, and $d$. Specifically, only individuals from Newfoundland and Labrador, Prince Edward Island, New Brunswick, Quebec, Ontario, Alberta, and the North West Territories - a total of $77.1 \%$ of the weighted sample - had been asked the questions in $b$ above; only individuals from Newfoundland and Labrador, Prince Edward Island, New Brunswick, some regions of Ontario and Saskatchewan, Alberta, Yukon, North West Territories and Nunavut - a total of $41.8 \%$ of the weighted sample - had been asked the questions in $c$ above; and only individuals from $\mathrm{New}$ Brunswick, some regions of Ontario, Saskatchewan, Alberta, North West Territories, and Nunavut - a total of $32.2 \%$ of the weighted sample - had been asked the questions in $d$ above.

The solution to estimating the three mental health categories involved a sequential process. First we estimated the prevalence of Diagnosed Mental Health Problems category from the full data. Then we estimated the prevalence of persons who met criterion $a$ for inclusion in the Undiagnosed Mental Health Problems category from the full data from the selfrated mental health question. From the group that had been administered both the question under criterion $a$ and the questions under criterion $b$, we estimated the proportion which met criterion $b$ but not criterion $a$. Similarly, from the set of persons administered all of the questions from $a, b$, and $c$, we estimated the proportion that met criterion $c$ without meeting criterion $a$ or $b$. This was repeated for the final set of questions, where the estimate was the proportion of persons meeting criterion $d$ without meeting criterion $a, b$ or $c$. These proportions were then added together to get a single prevalence estimate of persons in the Undiagnosed Mental Health Problems category.

There are two important aspects to note about this procedure. First, it makes the assumption that where the questions were not asked of all subjects, the sub-sample to which they were administered was nevertheless a representative one. And second, the proportion of persons that met each successive criterion is smaller, and therefore estimation errors due to sample bias should also be successively smaller at each stage.

Finally, the number of persons in the No Mental Health Problems category was estimated by subtraction. The results are presented in the first row of Table 1.

\section{Outcome Measures}

All outcome measures were estimated for each of the age-sex groups for all three Mental Health Problems categories defined above.

\section{Utilization}

Medical cost measures were derived from health care utilization during a one-year period measured as the number of all (i.e., not just mental health-oriented) general practitioner visits, specialist visits, and hospital days, as self-reported by each person in the survey. Use of physician services was determined based on the 
question which asked the number of times (excluding hospital stays) the person had seen/talked on the telephone with the health professional (general practitioner and specialist) about his/her physical, emotional or mental health in the past year. Use of hospital resources was determined based on questions about the number of nights spent in a facility. Utilization was estimated for each age-sex group for all three mental health categories.

\section{Unit Costs}

Unit costs of services for age-sex groups were based on Alberta provincial statistics provided by Alberta Health and Wellness (AHW) for the budget year 2000 to 2001, (15) adjusted for inflation and national levels, where appropriate. AHW maintains a database of physician claims which typically contains over 30 million records each year. The database records the location/type of service provided and the specialization of the physician for each claim. Average cost per visit to a general practitioner contained both the physician fee and the costs of laboratory tests. Specialist visits comprised visits made in specialists' offices and those made in hospital outpatient clinics (including emergency room). Average cost per specialist office visit included both the physician fee and the costs of diagnostic services. For specialist outpatient visits in a hospital setting, a hospital outpatient facility fee (based on the province-wide Alberta Ambulatory Care Classification System cost per visit) was added to the physician fee. Hospital inpatient stays included both facility and physician components. We used the province-wide per diem cost of $\$ 809$ ( $\$ 780$ in 2000 to 2001 adjusted by $6.5 \%$ for the change in the Consumer Price Index to 2003 to 2004) for a typical hospital day and added the average physician billing per day of hospitalization. Physician fees for Alberta were downward adjusted by $18 \%$ because Alberta's fees per service level were higher than the national average by this amount. (16) The unit costs of services according to age-sex groups were applied to each unit of service. Total medical cost per person is the sum of the costs for the three types of services.

\section{Work Loss - measurement and valuation}

Work loss due to long-term disability is estimated based on unemployment data in the past year. The proportion of people in each age-sex group who did not work throughout the year was obtained by tabulating answers to the question in the CCHS which asked each respondent for the number of weeks he/she worked at a job or business during the past year (including paid vacation leave, paid maternity leave, and paid sick leave). Lost work due to long-term disability (unemployment) was valued by an average annual wage, by sex, obtained from Statistics Canada [www.statcan.ca, Table 202-0102] for the year 2003. For 2003, the annual earnings were $\$ 25,300$ for women and $\$ 40,200$ for men.
In addition, work loss due to short-term disability (absenteeism) was also estimated based on questions that asked for the number of days spent in bed for all or most of the day (including hospitalization) in the 14-day period prior to the interview. The mean number of disability days in the two-week period for each age-sex group was multiplied by $5 / 7$ assuming that the disability days were distributed equally across weekdays and weekends and further multiplied by 26 weeks to obtain the annualized figure. This annualized figure was then multiplied by a daily wage obtained from Statistics Canada. The average weekly earnings for all industries was $\$ 690.57$ in 2003, averaged over fulltime and part-time workers. This figure was divided by 5 working days to obtain a daily figure of $\$ 138.11$ which was then applied to both men and women in each age-sex group.

TABLE 1

Unit values for outcomes by mental health status in Canada (2003) in population 20 and over

\begin{tabular}{|c|c|c|c|}
\hline & $\begin{array}{l}\text { No mental } \\
\text { illness }\end{array}$ & $\begin{array}{c}\text { Diagnosed } \\
\text { mental } \\
\text { illness }\end{array}$ & $\begin{array}{l}\text { Undiagnosed } \\
\text { mental } \\
\text { illness }\end{array}$ \\
\hline Frequency & 23261558 & 1877163 & 1605238 \\
\hline Percent total & $87.0 \%$ & $7.0 \%$ & $6.0 \%$ \\
\hline \multicolumn{4}{|l|}{ Male } \\
\hline 20 to 34 years & 3179784 & 150433 & 215549 \\
\hline 35 to 49 years & 3822703 & 235545 & 246683 \\
\hline 50 to 64 years & 2727415 & 195830 & 120105 \\
\hline $65+$ years & 1659625 & 71528 & 40779 \\
\hline \multicolumn{4}{|l|}{ Female } \\
\hline 20 to 34 years & 3154711 & 302591 & 332724 \\
\hline 35 to 49 years & 3840586 & 433365 & 396629 \\
\hline 50 to 64 years & 2751214 & 330160 & 190324 \\
\hline $65+$ years & 2125520 & 157711 & 62445 \\
\hline
\end{tabular}

Per person values

(weighted averages)

GP visits

2.9

Specialist visits

Hospital days

0.7

0.5

0.91

0.33

Percent not working (long-

term work loss

Disability days per year (shortterm work loss) 


\section{Health Utilities - measurement and valuation}

Health-related quality of life (HRQOL) for each person was assessed using the Health Utilities Index Mark 3 (HUI3). HUI3 is a preference-based, multi-attribute measure of HRQOL that uses a multiplicative utility function to assign valuations to different health states. ${ }^{(17,18)}$ In the HUI3 system, health status is defined by 8 attributes vision, hearing, speech, mobility, dexterity, emotion, cognition and pain. Each attribute has a number of different levels, and utility-based preference scores assigned to each attribute level are then combined multiplicatively to arrive at an overall utility score or index which measures the HRQOL associated with each individual's heath state. Overall HUI scores range in the CCHS Cycle 2.1 from -0.36 to 1.0 , with -0.36 representing the worst possible health state, 0.0 representing death and 1.0 representing full health. One year in full health is equivalent to one Quality Adjusted Life Year (QALY). According to Drummond, ${ }^{(7)}$ differences in overall utility scores of 0.03 are considered to be clinically significant.

In the CCHS, health status is an optional content questionnaire module which was only selected by 5 provinces - Newfoundland and Labrador, Prince Edward Island, Nova Scotia, New Brunswick and Quebec. As such, the analysis used sample weights of the 5 provinces to project HUI scores for the populations in the three mental health categories. To obtain a dollar value for the reductions in health utilities, the lost QALYs were multiplied by a figure of $\$ 50,000$, a dollar valuation commonly used in policy making. ${ }^{19}$

\section{Analysis Methods}

Our definition of economic burden focuses on the excess costs and losses in outcomes that are attributable to mental illness. It incorporates (1) the excess costs of the use of medical services resulting from mental illness, (2) the excess of work loss, both long term (unemployment) and short term (absenteeism), as a result of the disorder,
FIGURE 1

Classification of the components of economic burden

\begin{tabular}{|l|l|l|l|}
\hline \multirow{2}{*}{ Health care resources } & \multicolumn{2}{|l|}{ Direct (government) costs } & \multicolumn{2}{l|}{$\begin{array}{l}\text { Out-of-pocket and insurance } \\
\text { costs }\end{array}$} \\
\cline { 2 - 3 } Lost productivity & $\begin{array}{l}\text { Short-term } \\
\text { disability }\end{array}$ & $\begin{array}{l}\text { Long-term } \\
\text { disability }\end{array}$ & $\begin{array}{l}\text { Premature } \\
\text { mortality cost }\end{array}$ \\
\cline { 2 - 4 } Health outcomes & $\begin{array}{l}\text { Losses in health-related quality of life } \\
\text { from morbidity }\end{array}$ & \\
\hline
\end{tabular}

FIGURE 2

Cost per person by population characteristic (C\$)

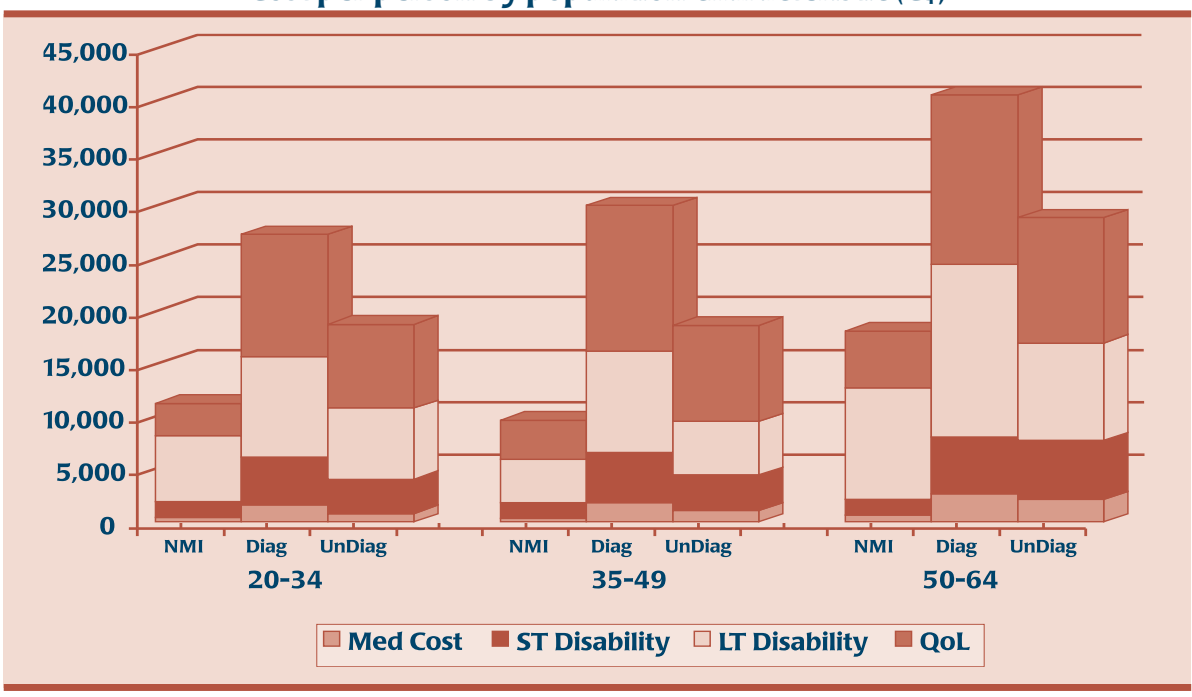

$\mathrm{N} \mathrm{MI}=$ no mental illness, Diag $=$ diagnosed mental illness, Un Diag $=$ undiagnosed mental illness, Med Cost $=$ direct medical cost, ST Disability = short-term disability, LT Disability = Long-term disability, QoL = quality of life.

TABLE 2

Economic burden of mental illness, Canada, 2003

\begin{tabular}{|c|c|c|c|c|}
\hline & $\begin{array}{l}\text { Diagnosed } \\
\text { (C\$\$m) }\end{array}$ & $\begin{array}{l}\text { Undiagnosed } \\
\text { (C\$\$m) }\end{array}$ & $\begin{array}{l}\text { Total burden } \\
\text { (C\$m) }\end{array}$ & $\begin{array}{l}\text { Per cent of } \\
\text { total }\end{array}$ \\
\hline Direct medical cost & 3,518 & 1,447 & 4,965 & 9.8 \\
\hline $\begin{array}{l}\text { Dollar value of work loss } \\
\text { (long-term) }\end{array}$ & 8,386 & 68 & 8,454 & 16.6 \\
\hline $\begin{array}{l}\text { Dollar value of work loss } \\
\text { (short-term) }\end{array}$ & 5,724 & 3,551 & 9,275 & 18.2 \\
\hline $\begin{array}{l}\text { Dollar value of loss in health } \\
\text { unit }\end{array}$ & 18,750 & 9,403 & 28,153 & 55.4 \\
\hline Total burden & 36,378 & 14,469 & 50,847 & $100.0 \%$ \\
\hline
\end{tabular}


and (3) the excess loss in health utilities attributable to mental illness. All three components are expressed in terms of the differences in these measures between the populations which have Mental Health Problems (Diagnosed and Undiagnosed) and the one with No Mental Health Problems.

Excess of or net medical costs due to mental illness were estimated in the way stated above for each age-sex group and aggregated to obtain the total excess cost for each mental health problem category. In the case of health utilities, non-mentally ill persons generally have higher HUI scores than mentally ill persons, diagnosed and undiagnosed (Table 1). Taking the differences in the scores gives us the losses or reductions in health utilities attributable to the disorder. Starting with the diagnosed category, we multiplied the losses in health utilities for each age-sex group by the number of persons in each sub-group to obtain the number of lost QALYs for each sub-group. The lost QALYs for all the agesex sub groups were then aggregated to obtain the total number of lost QALYs for the diagnosed category. The same was repeated for the undiagnosed category. The excess of income lost from long-term disability (work loss due to unemployment) in the past year and from short-term disability (work loss due to absenteeism annualized) were estimated in a similar way and added to the above costs to arrive at the total economic burden of diagnosed and undiagnosed mental illness population groups in Canada in 2003.

\section{Results}

The adult population of Canada, broken down by component, is shown in Table 1 . Of the total population, $7 \%$ or nearly 1.9 million people have been diagnosed with mental illness and a further $6 \%$ or 1.6 million people are undiagnosed. The largest portion of mentally ill individuals, for both males and females, falls in the 35 to 49 age range; with females having a higher prevalence rate (between 1.5 and 2 times more) in all age groups for both the diagnosed and undiagnosed categories.

Utilization of all health care services is highest for the diagnosed mentally ill, lowest for the non-mentally ill, with those in the undiagnosed category in the middle. The same is true for health utility and absenteeism. Employment is lower for the diagnosed, but more undiagnosed are working than those without mental illness. The dollar values of these estimates follow the physical measures, as shown in Figure 2 . The average medical cost per capita was $\$ 643$ for the non-mentally ill and $\$ 2,515$ for the diagnosed and $\$ 1,442$ for the undiagnosed.

On a per capita basis, the value of work loss (unemployment) is the highest component; this is the result of the high unit cost of an annual loss of employment (an average of $\$ 32,750$ per person).

The incremental economic burden of mental illness of persons over the age of 20 is shown in Table 2. The total burden for 2003 was about $\$ 51$ billion, with close to $30 \%$ of the cost incurred by the undiagnosed mentally ill population. Loss of health utilities, valued at $\$ 50,000$ per qualityadjusted life year (QALY), was by far the dominating effect, accounting for more than $50 \%$ of the total burden or around $\$ 28$ billion. The value of work loss from absenteeism (short-term disability) was about $10 \%$ higher than the value of work loss from unemployment (long-term disability); and together they account for about $35 \%$ of the burden. Medical expenses accounted for less than $10 \%$ of the total burden.

\section{Discussion}

We have constructed a comprehensive measure of the incremental economic burden of persons with mental illness aged 20 and above in Canada in 2003. Our total estimate of the burden was $\$ 51$ billion. The main components are shown in Table 2 , with the loss in health utilities accounting for more than one-half of the total burden.

Our results and analysis differ conceptually from those of Health Canada ${ }^{(3)}$ and Stevens and Joubert (SJ). ${ }^{(5)}$ Health Canada's analysis identified specific mental health services, while ours examines all health care services (used by persons with mental illness) for all causes, to measure direct health care costs. The Health Canada approach, how-ever, could not be used to identify the numbers of persons who received services. Health Canada conducted its estimate using a top-down approach, by which costs were assigned to mental health (and other conditions) according to how services were used; patients were not identified. Only services which were specifically coded (in billings and abstracts) to mental health were included. Further we included only hospital and medical services, where-as Health Canada included medicines as well. Both Health Canada and SJ, as well as our study, excluded community mental health. SJ used the Health Canada estimates for direct cost, but they used a similar approach to ours for indirect costs (i.e., excess costs as derived from the CCHS).

SJ have a definition of mental illness that includes depression and distress, while ours focused on diagnosed and undiagnosed mental illness. ${ }^{(5)}$ However, their definitions of both short- and long-term time-loss costs differ from ours. Our short-term time loss includes only time lost from work; SJ included time lost from all activities. We measure long-term costs as the value of the difference in employment between those with and without mental illness. SJ included only the inactivity days within the net time of unemployment. As we defined the entire loss of employment during a single year as our work-loss measure, our number is much larger than that of SJ.

One of the major benefits of our approach, as pointed out by $\mathrm{SJ},{ }^{(5)}$ is that resources used are traced to persons. This allows the overall burden to be assigned to a variety 
of specific risk factors, such as age, sex and socio-economic indicators, that is, a population health approach. A second benefit of our approach, which stems from the first, is that it allows us to estimate the net additional use of all healthcare resources, even if they have not been identified as "mental health" resources. Persons with mental illness may use more general health services, and this will be reflected in our analysis.

Our approach is also significant in identifying and including the undiagnosed mentally ill population (of around 1.6 million persons) in the cost estimate. More than one-fourth of the total burden was attributed to this population, and they accounted for about 30\% of the direct medical cost. Sareen, et al. ${ }^{(20)}$ have shown using the Mental Health Supplement of the Ontario Health Survey that in addition to the explicit criteria of an accepted diagnostic system, self-perceived need for mental health treatment provides valuable information for estimating the number of people in the population who need mental health services, and in devising public health strategies to minimize the burden of mental illness in Canada.

Several key assumptions should be highlighted, because the results are influenced by the approach taken. First, the health utility effect depends on the value placed on a QALY. It would have been substantial even if the valuation per QALY was very conservatively valued at $\$ 20,000$, as was suggested by Laupacis. ${ }^{(21)}$ In this case, the burden of HRQOL would still be over $\$ 11$ billion and nearly 35\% of the total burden. A value of $\$ 50,000$ placed on the loss of a QALY is the most widely used, but it is still arbitrary. If the value were $\$ 10,000$ lower/higher, this would deduct/add about $\$ 1,500$ to the difference per person. In any event, it is clear that HRQOL poses the major component of the economic burden of mental illness and cannot be ignored.

Second, the calculation of the annual loss placed on persons who are out of work in the long term depends on the perspective of the study. Using the current method, value lost is equal to lost wages for each "incremental" year of work lost. There is an alternative approach, the "friction cost" approach: if the worker is replaced by another who would, otherwise, not have been employed, there is an offset to the lost work. The societal measure of work loss is equal to the income lost by the disabled worker minus the gains made by the replacement worker. One method placed the friction cost estimate at about one-half that of the current method. ${ }^{(22)}$ If this were the case, long term losses would be closer to $\$ 9$ billion, rather than $\$ 16$ billion and the total burden would be reduced by this amount as well.

Our approach which is based on community survey has several limitations which will also influence our total estimate. Firstly, utilization estimates are based on personal recall, which are not as reliable as provider encounter records. However, a previous analysis using this data indicated that the estimate of global costs using this method is consistent with other methods. ${ }^{(15)}$

A second limitation is related to our use of unit costs. Our costs represent general health care costs, not those for mental health services. Per diem hospital costs, in particular, are lower for psychiatric admissions than they are for general admissions. In 2004 mental health inpatient services cost were roughly $\$ 600$ per day, compared to our estimate for general health services of $\$ 800$. Our estimate for hospital costs is somewhat higher, though some of these hospitalizations will be for non-mental health care and should be costed at the higher rate. We have no information what this percentage is.

A third and related, limitation is that we do not explicitly account for the contribution of physical comorbidities to the effects of mental illness on productivity. The literature indicates that the combination of physical and mental illness have a greater impact on disability. ${ }^{(23,24)}$ Using our approach to estimating undiagnosed cases, it would prove very difficult to estimate the net contribution of comorbidities.

A final limitation deals with the persons identified in the CCHS survey. As this was a household-based survey, it excluded persons with mental illness who were permanently institutionalized. According to estimates obtained from $\mathrm{CIHI}$, there were 403 long-term psychiatric beds in specialty psychiatric hospitals in 2003 to 2004 in Ontario. ${ }^{(25)}$ Assuming that Canadawide the beds to population ratio were the same as in Ontario, there would be 1,039 long-term residents in Canada. At 365 days per patient, the total bed days would be 379000 . At a very rough daily (Ontario) cost of between $\$ 300$ and $\$ 400$ for institutionalized mental health persons [Source: Alberta Mental Health Board] (\$109,500 - \$146,000 per person yearly), these institutionalized persons would cost between $\$ 113$ million and $\$ 151$ million. If this calculation is correct, then institutionalization costs would add about $3 \%$ to our estimates of direct costs.

Apart from the estimation and sampling errors, our investigation has left out mortality differences in indirect costs. Though they would be higher for the mentally ill population, these would likely not be very different between the groups. In direct costs, we have left out services of non-medical mental health professionals such as social workers and psychologists. This would add about 4 per cent to our estimate $\left(\mathrm{SJ}^{(5)}\right)$. The most important of these omissions are outpatient prescription drugs because they cannot be calculated from the CCHS. Health Canada ${ }^{(3)}$ estimates mental health drug costs to be $22 \%$ of total health care costs; this implies that the total direct costs with drugs would be about $\$ 6$ billion, up from about $\$ 5$ billion.

In conclusion, the economic burden of persons with mental illness as we have measured it is substantial. Losses in HRQOL, normally excluded, are substantial in this measure. While these are not costs (i.e., resource use items) as usually defined, they do impose hardship, and people are willing to pay to reduce the burden, i.e., they have economic value. However, it should be stressed that there are different ways of defining and measuring economic burden, and which one is appropriate depends on the purpose of the study. And finally, data have improved enormously in recent years, but while we are closer to a comprehensive estimate, 
better data in the area of institutional care, community care, and pharmaceuticals are needed to provide global estimates of cost that will be helpful to policy-makers.

\section{References}

1. Health Canada. A Report on Mental Illness in Canada. Ottawa: Health Canada, 2002. Cat. N. 0-662-328 17-5.

2. Standing Senate Committee on Social Affairs, Science and Technology (chaired by The Honourable Michael J. L. Kirby). Interim Report on Mental Health, Mental Illness, and Addiction: Reports 1, 2 and 3. Ottawa: Senate of Canada, November 2004.

3. Health Canada. Economic Burden of Illness in Canada, 1998. Ottawa: Health Canada, 2002. Cat. N. H21-136/1998E.

4. Moore R, Mao Y, Zhang J, Clarke K, Laboratory Centre for Disease Control. Economic Burden of Illness in Canada, 1993. Ottawa: Health Canada, 1997. Cat. N. H21-136/1993E.

5. Stephens $\mathrm{T}$, Joubert $\mathrm{N}$. The economic burden of mental problems in Canada. Chronic Dis Can 2001;22(1):1-10.

6. Phillips C. Health Economics. London: BMJ Books, 2005.

7. Drummond M. Introducing economic and quality of life measurements into clinical studies. Ann Med 2001;333:344-349.

8. Guyati G, Feeny D, Patrick D. Measuring health-related quality of life. Ann Intern Med 1993;118:622-729.

9. Schultz SE, Kopec JA. Impact of chronic conditions. Health Rep 2003;14(4):41-53.

10. Sainsbury Centre for Mental Health. The Economic and Social Costs of Mental Illness. London: The Sainsbury Centre, 2003.
11. Statistics Canada. Canadian Community Health Survey (Cycle 2.1). Public-Use Microdata File, 2003.

12. Starkes JM, Poulin CC, Kisely SR. Unmet need for the treatment of depression in Atlantic Canada. Can J Psychiatry 2005 Sep;50(10):580-90.

13. Robins LN, Wing J, Wittchen HU, Helzer JE, Babor TF, Burke J, et al. The Composite International Diagnostic Interview. An epidemiologic instrument suitable for use in conjunction with different diagnostic systems and in different cultures. Arch Gen Psychiatry 1998;45:10069-77.

14. Wittchen HU. Reliability and validity studies of the WHO-Composite International Diagnostic Interview (CIDI): A critical review. J Psychiatr Res 1994;28:57-84.

15. Ohinmaa A, Schopflocher D, Jacobs P, Golmohammadi, K, Demeter, S, Klarenbach, S. A population-based analysis of health behaviors, chronic diseases, and associated costs. Chronic Dis Can 2006;27(1):17-24.

16. Canadian Institute for Health Information. National Grouping System Categories Report, Canada 2002-3. Ottawa: Canadian Institute for Health Information, 2005.

17. Feeny D, Furlong W, Boyle M, Torrance GW. Multi-attribute health status classification systems: Health utilities index. Pharmacoeconomics 1995;7:490-502.

18. Feeny D, Furlong W, Torrance GW, et al. Multi-attribute and single-attribute utility functions for the Heath Utility Index Mark 3 System. Med Care 2002;40(2):113-128.
19. Hirth RA, Chernew ME, Miller E, Fendrick AM, Weissert WG. Willingness to pay for a quality-adjusted life year: In search of a standard. Med Decis Making 2000; 20(3):332-42.

20. Sareen, J, Stein MB, Campell DW, Hassard $\mathrm{T}$, Menec $\mathrm{V}$. The relation between perceived need for mental health treatment, DSM diagnosis, and quality of life: A Canadian population-based survey. Can J Psychiatry 2005;50(2):87-94

21. Laupacis A, Feeny D, Detsky AS, Tugwell PX. How attractive does a new technology have to be to warrant adoption and utilization? Tentative guidelines for using clinical and economic evaluations. CMAJ 1992;146(4):473-81.

22. Hutubessy RC, Van Tulder MW, Vondeling H. Annu Meet Int Soc Technol Assess Health Care. Int Soc Technol Assess Health Care Meet 1996;12:40.

23. Kessler RC, Ormel J, Demler O, et al. Comorbid mental disorders account for the role impairment of commonly occurring chronic physical disorders: Results from the National Comorbidity Survey. J Occup Environ Med 2003;45:1257-66.

24. Kessler RC, Frank RG. The impact of psychiatric disorders on work loss days. Psychol Med 1997;27:861-73.

25. Durbin J, Rush B, Smith B, Thibault K. The Impact Study Mid-Term Report 2007. Toronto: Health Systems Research and Consulting Unit, Centre for Addiction and Mental Health, 2007. 